\title{
Estudio preliminar del uso de un protocolo para evaluar el bienestar de vacas lecheras usando observaciones basadas en el animal ${ }^{\#}$
}

\author{
Preliminary study of the use of a protocol to assess the welfare of dairy cattle \\ using animal-based observations
}

\author{
C Arraño ${ }^{\mathrm{a}}$, A Báez ${ }^{\mathrm{b}}$, E Flor ${ }^{\mathrm{a}}$, H R Whayc, N Tadich ${ }^{\mathrm{a} *}$ \\ anstituto de Ciencias Clínicas Veterinarias, Facultad de Ciencias Veterinarias, Universidad Austral de Chile. \\ bInstituto de Estadística, Facultad de Ciencias, Universidad Austral de Chile. \\ 'Deparment of Clinical Veterinary Science, University of Bristol.
}

\begin{abstract}
SUMMARY
The aim of this study was to evaluate the welfare of dairy cows using a simplified protocol of animal-based observations. Twenty-two commercial dairy farms in the province of Valdivia, Chile, were visited once between March and July 2005. The farms were selected considering the willingness

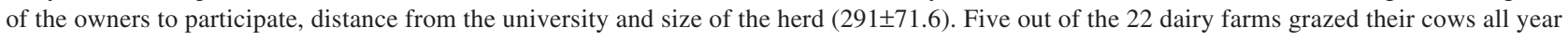
round; of the remaining 17 farms four of them kept the cows indoors all year round while 13 kept them indoors during the night. Visits started two hours before afternoon milking, and the cows were systematically observed at milking time in order to evaluate physical condition and behaviour. The data was entered into an Excel XP spread sheet. The results for each welfare indicator were grouped into five quintiles (A, top to E, bottom), then the dairy farms were ranked according to the number of indicators in each category. All the farms had at least one indicator in category A. Only one farm achieved five out of seven indicators in category A. The best evaluated indicator was body condition score of the cows, and the worse one was the "flight zone" of the cows. It can be concluded that no farm had consistenly good or bad results when compared to the others, with regards to evaluating cow welfare using animal based observations.
\end{abstract}

Palabras clave: bienestar animal, vacas lecheras.

Key words: animal welfare, dairy cows.

\section{INTRODUCCION}

El bienestar animal, su concepto (Fraser y Broom 1997, Webster 2001) y evaluación (Mench 2003, Whay y col 2003) han sido tema de investigación académica en los últimos años, con el objeto de desarrollar métodos objetivos para evaluarlo a nivel de granja, que permitan a los propietarios tomar medidas que lo mejoren, aumentando en forma indirecta la productividad de los animales (Main y col 2003, Mulleder y col 2003, Scott y col 2003, Whay col 2003).

Para evitar las pérdidas en producción y existencias, los bovinos de una lechería deben contar con un entorno que les permita crecer, madurar, reproducirse y mantener una buena salud (Blowey 1994). Por lo tanto, el éxito de la empresa lechera dependerá de la satisfacción de las necesidades básicas de los animales (Albright y Arave 1997). El término "necesidad" es usado para referirse a una deficiencia en un animal, la cual puede ser remedia-

\footnotetext{
Aceptado: 31.10.2006.

\# Financiado por proyectos FONDECYT 1040176 y 7040181.

* Casilla 567, Valdivia, Chile; ntadich@uach.cl
}

da por la obtención de un recurso en particular o respondiendo a un estímulo corporal o ambiental (Fraser y Broom 1997). De hecho, las bases para determinar las necesidades de los animales se deducen a menudo de situaciones en las cuales hay alguna alteración en su ambiente (Albright y Arave 1997). Los individuos pueden tener una variedad de necesidades, algunas de mayor urgencia que otras, y cada una es consecuencia de la biología del animal; en general, se distinguen necesidades fisiológicas y de comportamiento (Fraser y Broom 1997).

Un animal que no se encuentre en un estado de bienestar no va a desarrollar todo su potencial productivo. Según Grandin (2000), los ganaderos pierden dinero cotidianamente por el maltrato que se tolera en el campo simplemente porque no lo perciben como un problema, $\mathrm{y}$, por ende, no le buscan solución.

A pesar de que existe un debate acerca de la definición del término bienestar, su evaluación puede ser categorizada, en general, en mediciones basadas en el animal y aquellas basadas en el recurso. Las medidas basadas en el animal, tales como condición corporal, estado de enfermedad, grados de lesiones y zona de fuga, dan una indicación del desempeño/resultado del sistema de crianza (Main y col 2003 ${ }^{\mathrm{a}}$ ). Para que una herramienta apropiada de evaluación de bienestar pueda ser usada en 
lecherías se requiere que las mediciones sean válidas, confiables y posibles de realizar (Mülleder y col 2003).

Las mediciones basadas en el animal que toman importancia a la hora de medir el bienestar son:

Las que miden condición física del animal: como la condición corporal, la cual es utilizada para estimar las reservas energéticas del animal (Edmonson y col 1989), conformación de las pezuñas, limpieza del pelaje, alopecias, presencia de heridas, entre otras (Whay y col 2003).

Las que se refieren al comportamiento del animal: por ejemplo, zona de fuga, agresividad, interacción social, actividad ruminal, por mencionar algunas (Molony y Kent 1997, Whay y col 2003).

Indicadores sanguíneos de estrés: entre los más comúnmente utilizados se encuentran las concentraciones de cortisol, glucosa, prolactina, hematocrito, leucocitos y proteínas de la fase aguda como la haptoglobina (Tadich y col 2003).

El consumidor moderno considera inaceptables los sistemas de producción animal cuando afectan el bienestar de los animales en algún punto de la cadena productiva (Seng y Laporte 2005), exigiendo más información y transparencia, con etiquetas que certifiquen la trazabilidad del producto, indicando los métodos de crianza, los cuidados aplicados y el uso de alimentos inocuos (Littin y Mellor 2005). Esto ha llevado a un aumento de las exigencias legales y reglamentarias en torno al bienestar animal (Gallo y Tadich 2004).

Chile, con el ingreso al comercio internacional, ha incorporado el concepto de Buenas Prácticas Ganaderas, el cual se emplea en el programa PABCO (Planteles Bovinos Bajo Control Oficial), supervisado por el Servicio Agrícola y Ganadero; este programa pretende fortalecer la competitividad de los planteles insertos en él a través de medidas de prevención, erradicación y control de enfermedades prevalentes y fomento del uso de Buenas Prácticas Ganaderas (Chile 2002).

Este estudio es una investigación preliminar en el uso de un protocolo simplificado de mediciones basadas en el animal que permitirían evaluar el bienestar de vacas de lecherías.

\section{MATERIAL Y METODOS}

Se utilizaron 22 lecherías ubicadas en la provincia de Valdivia que aplicaban como método de manejo la estabulación parcial, estabulación permanente o a praderas. Estas fueron seleccionadas por conveniencia tomando en cuenta la disposición del propietario para participar del estudio, su facilidad de acceso y el tamaño del rebaño. Se visitaron por una única vez entre marzo y junio del 2005. La obtención de la información fue realizada siempre por la misma persona.
Para evaluar el bienestar se utilizó el protocolo descrito por Whay y col (2003), simplificado para las condiciones chilenas. Se utilizó siempre el mismo evaluador, masculino, complexión mediana, y siempre utilizó la misma vestimenta consistente en overall de color azul petróleo y botas de caucho negro. De esta forma se evaluaron los aspectos relacionados con comportamiento y condición física de los animales que se describen a continuación.

Zona de Fuga. La "zona de fuga" de las vacas corresponde al espacio que el animal considera como propio (Whay y col 2003). Esto se determinó al azar en un 5\% de las vacas, en el patio de espera antes de que las vacas entraran a la ordeña. El examinador se acercó a las vacas lentamente (un paso cada 2 segundos) en un ángulo de $90^{\circ}$, hasta que el animal doblaba el cuello para alejarse o se alejaba efectivamente, para luego estimar la distancia que la vaca permitió acercársele con una varilla de PVC con una longitud de $2,5 \mathrm{~m}$, graduada cada $50 \mathrm{~cm}$.

Condición física. A medida que las vacas iban saliendo de la sala de ordeña hacia el patio de salida, se observó al $20 \%$ de las vacas en ordeña por medio de un muestreo sistemático, en que una de cada cinco vacas fue escogida para evaluar los siguientes aspectos:

Estado de nutrición: dado por la condición corporal medida en una escala de 1 a 5 (Edmonson y col 1989).

Evaluación de la locomoción: otorgando un puntaje de 0 (vaca sana) a 4 (vaca que prefiere evitar el movimiento) (Tadich y col 2005).

Presencia de lesiones: en la zona de los tarsos, lesiones en la grupa (glúteos, alas del íleon, isquion y zona perimetral de la cola, de un mínimo de $5 \mathrm{~cm}$ de diámetro), alopecias en la grupa (un mínimo de $5 \mathrm{~cm}$ de diámetro).

Grado de suciedad de los animales, grado 1: suciedad hasta la altura de los tarsos, grado 2: suciedad sobre la rodilla, grado 3: patas, ubres y flanco sucio (Hugues 2001).

\section{ANALISIS ESTADISTICO}

Los antecedentes recolectados fueron incorporados a una base de datos en una planilla electrónica del programa computacional Microsoft ${ }^{\circledR}$ Excel XP. En el caso de los índices que evaluaban el grado de locomoción, la condición corporal (CC) y el grado de suciedad, se ingresaron los puntajes que representaban una alteración del bienestar de las vacas; así, ingresaron a las categorías, los porcentajes de vacas que tuvieran un grado de locomoción $\geq$ 2 , vacas con $\mathrm{CC}<2$, y suciedad de grado 3 . Los porcentajes para cada uno de los indicadores se dividieron en categorías. Para definir el número de categorías, se calculó el número de intervalos (m) (Whay y col 2003).

$\mathrm{m}=\sqrt{ } \mathrm{n}$ donde $\mathrm{n}=$ número de lecherías. De esta forma se obtuvieron 5 categorías (A, B, C, D, E). 
Una vez obtenido el número de categorías, se calculó el recorrido $(\mathrm{R})$ del indicador, que es la diferencia entre el porcentaje máximo y el mínimo encontrado para éste $(\mathrm{X})$, para luego calcular la amplitud de la categoría (A) al dividir el recorrido $(\mathrm{R})$ por el número de intervalos $(\mathrm{m})$ :

\section{RESULTADOS Y DISCUSION}

Las 22 lecherías presentaron un tamaño promedio de $291 \pm 71,6$ (rango 185-460) vacas en ordeña al momento de la visita.

De las 22 lecherías, cinco $(22,7 \%)$ mantenían sus vacas a pastoreo todo el año, cuatro $(18,2 \%)$ mantenían las vacas estabuladas todo el año y $13(59,1 \%)$ estabulaban las vacas durante el invierno. El sistema de estabulación en aquellas lecherías que estabulaban era en base a cubículos.

Todas las lecherías visitadas tenían vacas con grados de cojera $\geq 2$ ( $\min .4 \%$, $\max .52,8 \%$ ). Sólo cinco lecherías tuvieron porcentajes de cojeras entre un 3,6\% y un $13,6 \%$, finalizando en la categoría A. Acuña y col (2004) indican que cuando el porcentaje de vacas cojas supera el $15 \%$, se puede considerar como un problema de rebaño. En este estudio, todas las lecherías ubicadas en las categorías B a la E tendrían problemas de cojeras (17 lecherías). La media fue de $24,1 \%$ de vacas cojas en las 22 lecherías. Los datos sobre prevalencia de cojeras que existen en la literatura en Chile son variados y van desde un $9 \%$ hasta un $32 \%$ (Tadich y col 2005, Flor 2006).

Whay y col (2003) señalan que los ganaderos subvaloran la presencia de vacas cojas en los rebaños, ya que no existe relación entre los estudios de prevalencia de cojeras que se realizan en terreno y los registros de tratamientos existentes en las lecherías, lo que implica que en la mayoría de los casos las cojeras no son tratadas o no son diagnosticadas, a pesar de ser consideradas como uno de los factores más importantes a la hora de evaluar el bienestar de las vacas lecheras, pues se relacionan con el dolor que sufre el animal y la alteración de su comportamiento natural. El problema de las cojeras provoca consecuencias negativas en el bienestar animal y en la rentabilidad del sistema (Warnick y col 2001, Zimmerman 2001, Galindo y Broom 2002, Green y col 2003).

$\mathrm{Al}$ observar el porcentaje de vacas con $\mathrm{CC}<2,0$, la media encontrada fue de un $4,8 \%$ de vacas con esa condición. Contreras (1998) señala que las vacas al parto deberían llegar con una CC de 3,0-3,5 y que la disminución en los primeros 70 días de lactancia no debería ser superior a un punto e idealmente 0,5 , como pérdida máxima, para iniciar la recuperación de las reservas energéticas a los 80-90 días posparto. Al distribuir las lecherías en las cinco categorías para este indicador (cuadro 2), 17 lecherías $(77,2 \%)$ se encontraron en la categoría A; por lo que la mayoría de ellas tenía menos de un $5 \%$ de vacas con $\mathrm{CC}<2,0$, siendo el indicador que observó el mejor comportamiento en las lecherías estudiadas. La CC es considerada por distintos autores como un buen predictor tanto del desempeño reproductivo como de la salud de las vacas (Waltner y col 1993, Hady y col 1994). Waltner y col (1993), Buckley y col (2003) y Shrestha y col (2005) indican que a medida que disminuye la CC disminuyen los índices reproductivos y se presentan metritis con mayor frecuencia en los primeros 20 días posparto. Además, Waltner y col (1993) señalan que aquellas vacas que pierden más de 1,5 puntos de CC posparto, disminuyen su producción potencial al compararlas con aquellas que no disminuyen su CC.

En la figura 1 se puede observar que en cuatro lecherías $(18,2 \%)$, las vacas aceptaban que el examinador se acercara a una distancia de $1 \mathrm{~m}$, mientras que en 10 lecherías $(45,4 \%)$ la zona de fuga fue $\geq$ a $2,0 \mathrm{~m}$. Si bien, la literatura no describe cuál es la zona de fuga aceptada como adecuada, estos resultados son similares a los encontrados por Whay y col (2003), en vacas de lecherías del Reino Unido, la cual osciló entre 0,7 y 3,2 m. La zona de fuga es considerada por Mülleder y col (2003)

Cuadro 1. Distribución de las cinco categorías (A-B-C-D-E) para los indicadores de bienestar observados en las vacas. Distribution of the five categories (A-B-C-D-E) for the different indicators of welfare observed in the cows.

\begin{tabular}{lccccc}
\hline Observación/Categorías & A & B & C & D & E \\
\hline Vacas con grado de cojera $\geq 2(\%)$ & $3,6-13,6^{*}$ & $13,7-23,6$ & $23,7-33,6$ & $33,7-43,6$ & $43,7-53,6$ \\
Vacas con condición corporal $<2(\%)$ & $0-5$ & $5,1-10$ & $10,1-15$ & $15,1-20$ & $20,1-25$ \\
Zona de fuga (m) & $0,7-1,1$ & $1,2-1,5$ & $1,6-1,9$ & $2,0-2,4$ & $2,5-2,8$ \\
Vacas con lesiones tarso (\%) & $0-2$ & $2,1-4$ & $4,1-6$ & $6,1-8$ & $8,1-10$ \\
Vacas con lesiones grupa (\%) & $0-1,6$ & $1,7-3,2$ & $3,3-4,8$ & $4,9-6,4$ & $6,5-8$ \\
Vacas con alopecias grupa (\%) & $0-2,3$ & $2,4-4,6$ & $4,7-6,9$ & $7,0-9,2$ & $9,3-11,8$ \\
Vacas con suciedad en flancos $(\%)$ & $6,4-24,4$ & $24,5-42,4$ & $42,5-60,4$ & $60,5-78,4$ & $78,5-96,4$ \\
\hline
\end{tabular}

* Los números en los casilleros indican el rango porcentual en que se encuentra cada categoría. 
Cuadro 2. Distribución de las 22 lecherías en las cinco categorías (A-B-C-D-E) según los indicadores usados para evaluar el bienestar.

Distribution of the 22 dairy farms in the five categories (A-B-C-D-E) regarding the different indicators used to evaluate welfare.

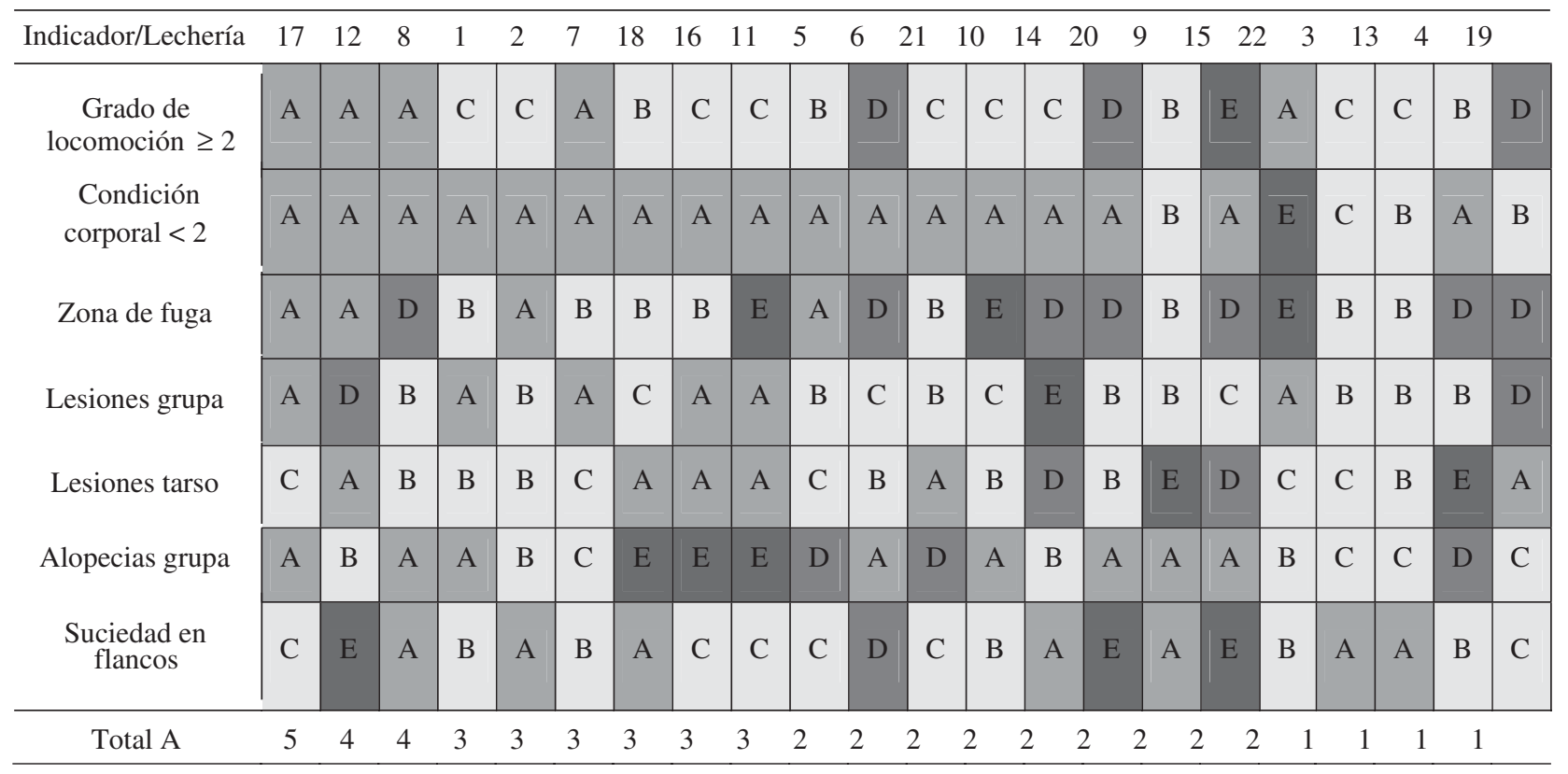

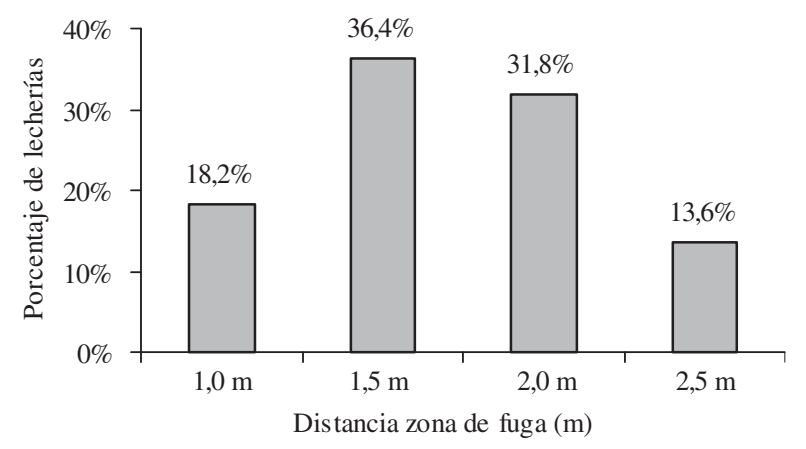

Figura 1. Distribución porcentual de las 22 lecherías en relación a la zona de fuga (m) de sus vacas. (m) of their cows.

Distribution of the 22 dairy farms regarding the flight zone

como un indicador importante de la relación hombreanimal y permite observar cómo reaccionan las vacas frente a la presencia de personas. De los siete indicadores de bienestar evaluados, la zona de fuga fue la que observó el peor comportamiento, con siete lecherías en categoría D y tres en categoría E (45,5\% del total) (cuadro 2 ). Sin embargo, para poder obtener conclusiones más confiables sería necesario aumentar el número de vacas evaluadas (Waiblinger y Menke 2003).

Según Grandin (2000), la zona de fuga está determinada por la docilidad o la rusticidad del animal y lo acostumbrado que esté el ganado a la presencia del hombre, siendo afectada por experiencias estresantes previas. Un animal que pasa por una situación de peligro aprende a ser más cauto en el futuro y aumenta su distancia de zona de fuga. Las vacas que son manejadas de forma tranquila, con arreos que no alteren su comportamiento y sin apuros, permiten que una persona se acerque hasta incluso poder tocarlas, a diferencia de las vacas que han sido manejadas bruscamente, las cuales tienen una zona de fuga mucho mayor. Por otro lado, vacas que son manejadas en estabulación con un contacto cercano con personas deberían tener una menor zona de fuga que aquellas vacas que se mantienen a pradera. Cabe señalar que en este estudio una de las lecherías que mantenía a los animales a pradera se ubicó en la categoría A. Las cojeras podrían interferir con la medición de la zona de fuga, ya que estos animales evitarían el movimiento por el dolor (Whay y col 2003). Sin embargo, Mülleder y col (2003) comprobaron que no existe una correlación negativa entre el grado de cojera y la distancia de la zona de fuga.

En 18 lecherías se detectaron vacas con lesiones en los tarsos. El porcentaje de vacas con lesiones varió desde un $0 \%$ a un $9,68 \%$, con una media de $3,53 \%$. Al categorizar los porcentajes de vacas con lesión en los tarsos por lechería, el 59\% de las lecherías (n=13) se ubicó en las categorías A y B, es decir esas lecherías tuvieron porcentajes de presentación desde un $0 \%$ a un $6 \%$, lo cual es similar a lo encontrado por Whay (2003) en el Reino Unido. Las lesiones de tarso pueden ser causadas debido a un mal diseño de las instalaciones, así como también por una condición sanitaria deficiente de las mismas. En los sistemas de estabulación en base a cubículos, el uso de hormigón sin una superficie de amortiguación provoca traumatismos en las zonas de poca musculatura y abrasiones en corvejones y codos de los 


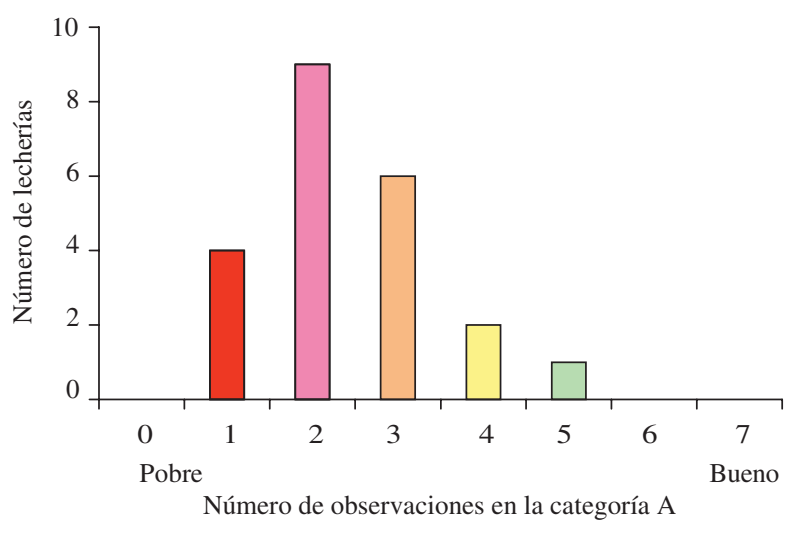

Figura 2. Distribución de las lecherías en relación al número de indicadores que se encontraron en la categoría A.

Distribution of the dairy farms regarding the number of indicators found in the category A.

animales (Hughes 2001). De acuerdo con Whay y col (2003), existe una correlación positiva entre la presencia de lesiones en esa zona y las cojeras y subsecuentemente sobre el bienestar animal.

En 18 de las 22 lecherías se observaron vacas con lesiones en la grupa. En una lechería se observó un 7,5\% de vacas con lesión en la grupa, la media fue $2,8 \%$. Las lesiones de grupa variaron desde un $0 \%$ a casi un $8 \%$ de presentación. Un $68,2 \%$ de las lecherías estuvo en las categorías A y B (cuadro 2). Esto indicaría que no es un problema muy habitual en las lecherías visitadas y podrían ser consideradas como hallazgos. Sin embargo, las molestias y el rascado pueden interferir con el descanso y la alimentación normal; por otro lado, existen algunos riesgos para la salud del animal, como es el caso de una herida penetrante, la cual puede ser provocada, por ejemplo, por el uso de picanas o la administración de productos farmacéuticos mediante el uso de inyecciones.

En 21 de las 22 lecherías se observaron alopecias en la grupa de las vacas, con un rango que varió desde un $0 \%$ a $10,45 \%$, la media fue de un $4,5 \%$. Entre las causas de alopecias, se describen el proceso de cicatrización que sucede a las heridas cutáneas profundas que destruyen folículos, alteraciones hereditarias y congénitas, alteraciones metabólicas, alopecia traumática por rascado asociada con infestaciones de piojos, garrapatas u otros ácaros, fricción con puertas de los pasillos, dermatitis causadas por intoxicaciones y dermatitis micótica (Radostits y col 2002). Es necesario definir un tamaño mínimo para las alopecias, que en este caso fue de $5 \mathrm{~cm}$ de diámetro como mínimo, ya que pueden existir "marcas" que podrían confundir al observador, como es por ejemplo, las que deja el toro en la zona de la grupa al momento de la monta.

Las 22 lecherías presentaron vacas con suciedad en los flancos y las frecuencias de presentación variaron desde un $6 \%$ hasta más del $90 \%$ de las vacas con los flancos sucios. Al clasificarlas por categorías, 15 lecherías $(68,2 \%)$ tenían sobre un $25 \%$ de las vacas observadas sucias, por lo que se encontraron entre las categorías B y E. Hugues (2001) y Whay y col (2003) señalan que esto se debería a que las personas que trabajan en las lecherías desarrollan cierta tolerancia a la situación. Esto puede llevar a una gran exposición a patógenos ambientales, como E. coli y Streptococcus uberis, que pueden provocar mastitis ambientales (Bradley y Green 2000). El hecho de que exista un alto número de vacas con la glándula mamaria sucia aumenta el tiempo de preparación que las vacas necesitan antes de la ordeña, lo cual demora el proceso en general y hace que las vacas tengan que pasar más tiempo de pie en el patio de espera, sin alimento y con una alta densidad de animales en un espacio reducido (Hugues 2001).

Una de las principales causas de suciedad en las vacas, es la diarrea. Hugues (2001) argumenta que existe una relación entre la consistencia del material fecal y el grado de suciedad que tienen los animales. El otorgar un puntaje a la suciedad puede ayudar a identificar las fuentes de contaminación. En el caso de las patas sucias, se puede deber al material fecal que queda en los pasillos por donde transitan los animales; una ubre sucia puede reflejar el estado de las camas. Los flancos sucios de las vacas puede deberse al llamado "efecto pincel" que se produce cuando las colas sucias, por las heces que se adhieren, se mueven y arrastran el material fecal por la zona perineal y hasta los flancos. El mejorar la limpieza de los lugares por donde transitan y duermen los animales puede producir una mejora significativa en limpieza de las vacas, evitando los factores que alteren su bienestar (Hugues 2001).

El cuadro 2 y figura 1 presentan la distribución de los indicadores de bienestar animal evaluados en las 5 categorías, por lechería; es así que la lechería 17 fue la que obtuvo el mayor número de indicadores en la categoría A (5 de 7), mientras que la lechería 19 fue la que presentó un solo indicador en la categoría A, junto con 3 en la categoría D. La CC fue el indicador que observó el mejor comportamiento en las lecherías, mientras que la zona de fuga fue el indicador que observó el peor comportamiento. Esta categorización en base a indicadores de bienestar permite comparar entre lecherías, lo que podría significar un incentivo hacia los ganaderos para mejorar sus sistemas (Main y col 2003 ${ }^{\text {b }}$. En general, en cada lechería los indicadores de bienestar evaluados presentaron una amplia distribución en las cinco categorías; ninguna lechería tuvo resultados consistentemente buenos o malos en relación a las demás (figura 2). En cada una hubo fortalezas y debilidades en diversas variables. De acuerdo con Whay y col (2003), es muy difícil que los ganaderos puedan producir consistentemente altos índices de bienestar en todos los aspectos, lo que implica que las intervenciones para mejorar el bienestar de las vacas de- 
ben estar dirigidas a los problemas específicos de cada lechería.

Las condiciones observadas en estas 22 lecherías no necesariamente representan la condición de las lecherías de la provincia o de la Décima Región. Este estudio preliminar es un aporte para fijar criterios con los cuales poder evaluar el bienestar animal a través de la observación del estado físico y el comportamiento de los animales. Las cojeras de las vacas, CC y la zona de fuga, entre otros, han sido considerados por Whay y col (2003), Main y col (2003b) y Fraser (2003) como los factores importantes en la determinación del bienestar de las vacas lecheras y deben ser tomadas en consideración. El determinar parámetros objetivos de observación es considerado como un método adecuado para poder evaluar el bienestar de las vacas en lecherías por diversos autores y así hacer comparables los resultados (Main y col 2003 ${ }^{\mathrm{b}}$, Whay y col 2003). Para poder definir mejor el protocolo a utilizar se deben incluir otros parámetros, como comportamiento social de las vacas, aumentando el número de lecherías y categorizándolas de acuerdo a sus características tecnológicas y productivas. También sería importante establecer las características del observador en cuanto a su género, vestimenta y tamaño corporal.

\section{RESUMEN}

El objetivo del presente estudio fue evaluar el bienestar de vacas de lechería usando un protocolo simplificado de observaciones basadas en el animal. Las 22 lecherías se visitaron por una sola vez entre marzo y junio del 2005. Las lecherías fueron seleccionadas por conveniencia, tomando en cuenta la disposición del propietario para participar, su facilidad de acceso y el tamaño del rebaño $(291 \pm 71,6)$. De las 22 lecherías, 5 de ellas mantenían a sus vacas a pradera; de las 17 restantes, 4 tenían estabulación permanente y 13 las mantenían estabuladas durante la noche. La visita comenzó dos horas antes de la ordeña de la tarde; durante la visita los animales fueron observados sistemáticamente al momento de la ordeña, para evaluar la condición física y el comportamiento. Las observaciones recopiladas fueron ingresadas a una planilla electrónica Microsoft ${ }^{\circledR}$ Excel XP. Los resultados encontrados para las indicadores de bienestar basados en observaciones de los animales se agruparon en cinco categorías (A: lo mejor/ E: lo peor), luego las granjas fueron ordenadas de acuerdo al porcentaje de indicadores en cada categoría. Las 22 lecherías tuvieron al menos un indicador en la categoría A. Una sola lechería logró tener cinco indicadores de los siete observados, en la categoría A. El indicador mejor evaluado fue la condición corporal, y el peor evaluado fue la "zona de fuga" de las vacas. Se concluye que ninguna lechería tuvo resultados consistentemente buenos o malos en relación a las demás, al evaluar el bienestar de las vacas mediante observaciones basadas en el animal.

\section{REFERENCIAS}

Acuña R, D Alza, J Junqueira, K Nordlund, J Ramos. 2004. Cojeras del bovino. Intermédica, Buenos Aires.

Albright J C, C Arave. 1997. Behavioural responses to management systems. In: The Behaviour of Cattle. CAB International, Oxon, UK, Pp 127-153.

Blowey R. 1994. Comparative climatic requirements. In: Livestock Housing. CAB International, Cambridge, UK.
Bradley A J, M Green. 2000. A study of the incidence and significance of intramammary enterobacterial infections acquired during the dry period. J Dairy Sci 83, 1957-1965.

Buckley F, K O'Sullivan, J F Mee, R D Evans, P Dillon. 2003. Relationship among milk yield, body condition, cow weight and reproduction in spring-calved Holstein Friesians. J Dairy Sci 86, 2308-2319.

Chile, 1997. Instituto Nacional de Estadísticas. INE. VI Censo Nacional Agropecuario.

Chile, 2002. Ministerio de Agricultura. Servicio Agrícola y Ganadero. Planteles Bovinos Bajo Control Oficial. Manual de Procedimientos. Protección Pecuaria Inspección y Certificación.

Contreras, P. 1998. Síndrome de movilización grasa en vacas lecheras al inicio de la lactancia y sus efectos en salud y producción de los rebaños. Arch Med Vet 30, 17-27.

Edmonson A J, I J Lean, L Weaver, T Farver, G Webster. 1989. A body condition scoring chart for Holstein dairy cows. J Dairy Sci $72,68-78$.

Flor E. 2006. Claudicaciones en vacas de rebaños lecheros de la Décima Región, Chile: Prevalencia, lesiones y factores de riesgo. Tesis de Magíster en Ciencias, Universidad Austral de Chile.

Fraser A J, D Broom. 1997. Welfare terminology and concepts. In: Farm Animal Behavior and Welfare. $3^{\text {rd }}$ ed. CABI Publishing, Oxon, UK, Pp 256-357.

Fraser D C. 2003. Assessing animal welfare at the farm and group level: The interplay of science and values. Animal Welfare 12, 433-443.

Galindo F, D Broom. 2002. Effects of lameness of dairy cows. J Appl Anim Welf Sci 5, 193-201.

Gallo C, N Tadich. 2004. Bienestar Animal y calidad de carne durante los manejos previos al faenamiento en bovinos. Resúmenes Seminario "Producción Animal de Calidad Contemplando Bienestar Animal”, Valdivia, Chile, Pp 41-55.

Grandin T. 2000. Livestock handling and transport. CABI, Wallingford, UK.

Green L, V J Hedges, Y H Schukken, R W Blowey, AJ Packington. 2002. The impact of clinical lameness on the milk yield of dairy cows. J Dairy Sci 85, 22150-2256.

Hady P J, J Domecq, J Kaneene. 1994. Frequency and precision of body condition scoring in dairy cattle. J Dairy Sci 77, 1543-1547.

Hugues J. 2001. A system for assessing cow cleanliness. In Practice 23, 517-524.

Littin K E, D Mellor. 2005. Strategic animal welfare issues: ethical and animal welfare issues arising from the killing of wildlife for disease control and environmental reasons. Rev Sci Tech 24, 767-782.

Main D C, J Kent, F Wemelsfelder, E Ofner, F Tuyttens. 2003 Applications for methods of on-farm welfare assessment. Animal Welfare 12, 523-528.

Main D C, H R Whay, L E Green, A J F Webster. 2003 ${ }^{\text {b }}$. Effect of the RSPCA freedom food scheme on the welfare of dairy cattle. Vet Rec 153, 227-231.

Mench J A. 2003. Assessing animal welfare at the farm and group level: A United States perspective. Animal Welfare 12, 493-503.

Molony V, J Kent. 1997. Assessment of acute pain in farm animals using behavioural and physiological measurements. J Anim Sci 75, 266-272.

Mülleder C, J Troxler, S Waiblinger. 2003. Methodological aspects for the assessment of social behaviour and avoidance distance on dairy farms. Animal Welfare 12, 579-584.

Radostits O M, C Gay, D Blood, H Kenneth. 2002. Dolor. En: Medicina Veterinaria. Tratado de las enfermedades del ganado bovino, ovino, porcino, caprino y equino. $9^{\mathrm{a}} \mathrm{ed}$. McGraw-Hill Interamericana, Madrid, España.

Scott E M, J L Fitzpatrick, A M Nolan, J Reid, M L Wiseman. 2003. Evaluation of welfare state based on interpretation of multiple indices. Animal Welfare 12, 457-468.

Seng P M, R Laporte. 2005. Animal welfare: the role and perspectives of the meat and livestock sector. Rev Sci Tech 24, 613-623. 
Shrestha H K, T Nakao, T Suzuki, M Akita, T Higaki. 2005. Relationships between body condition score, body weight, and some nutritional parameters in plasma and resumption of ovarian cyclicity postpartum during pre-service period in high-producing dairy cows in a subtropical region in Japan. Theriogenology 64, 855-866.

Tadich N, C Gallo, R Echeverría, G Van Schaik. 2003. Efecto del ayuno durante dos tiempos de confinamiento y de transporte terrestre sobre algunas variables sanguíneas indicadoras de estrés en novillos. Arch Med Vet 35, 171-185.

Tadich N, E Hettich, G Van Schaik. 2005. Prevalencia de cojeras en vacas de 50 rebaños lecheros del sur de Chile. Arch Med Vet 37, 29-36.

Waiblinger S, C Menke. 2003. Influence of a sample size and experimenter on reliability of measures of avoidance distance in dairy cows. Animal Welfare 12, 585-589.
Waltner S S, J McNamara, J Hillers. 1993. Relationships of body condition score to production variables in high producing Holstein Dairy Cattle. J Dairy Sci 76, 3410-3419.

Warnick L, D Janssen, C Guard, Y Gröhn. 2001. The effect of lameness on milk production in dairy cows. J Dairy Sci 84, 1988-1997.

Webster A J F. 2001. Farm animal welfare: The five freedoms and the free market. Vet $J 161,229-237$.

Whay H R, D Main, L Green, A J F Webster. 2003. Assessment of the welfare of dairy cattle using animal-based measurements: direct observations and investigation of farm records. Vet Rec 153, 197-202

Zimmerman A. 2001. Lameness in Dairy Cattle: Are Activity levels, Hoof Lesions and Lameness Correlated? Thesis Master of Science, University of British Columbia, Canada. 
